\title{
化工单元操作中理论板与传质单元数之间关系的探究
}

\author{
李学慧, 陈梁, 吴正舜* \\ 华中师范大学国家级化学实验示范中心, 武汉 430079
}

摘要: 填料塔与板式塔是化工单元操作过程中的常见设备, 而填料塔中填料层高度的计算与板式塔中理论板数的确定是 关键, 教材中有关填料层高度的计算采用的是传质单元数法, 而板式塔中理论板的确定采用是McCable-Thiele方法来确 定的, 有关传质单元数与理论板它们之间的关系介绍的较少。本文采用吸收因子法详细介绍了理论板数与传质单元数的 求法, 并由此建立了它们之间的定量关系, 可用于二类塔的比较, 对于填料塔的分析与设计具有一定的指导作用, 有助 于对吸收与精馏单元操作过程的学习与理解。

关键词: 化工单元操作; 理论板; 传质单元数; 吸收因子

中图分类号: G64; 06

\section{Study on the Relationship between the Theoretical Plate Number and the Mass Transfer Unit Number in Chemical Unit Operation}

\author{
Xuehui Li, Liang Chen, Zhengshun Wu * \\ National Demonstration Center for Experimental Chemistry Education, Central China Normal University, Wuhan 430079, \\ P. R. China.
}

\begin{abstract}
Packed tower and plate tower were common equipment in the operation of chemical units. The calculation of the height of the packing layer in the packed tower and the determination of the number of theoretical plates in the tray tower were the key. The calculation of the height of the packing layer in the textbook was based on the mass transfer unit, and the determination of the theoretical plate in the plate tower was determined by the McCable-Thiele method, and the relationship between the number of mass transfer units and the theoretical plate was less. In this paper, The mass transfer unit number and the theoretical plate number were respectively calculated by the absorption factor method in detail, and the quantitative relationship between them was established. It could be used for the comparison of the two type towers, which had a certain guiding role for the analysis and design of the tower, and helped students to learn and understand the process of absorption and rectification unit operations.
\end{abstract}

Key Words: Chemical unit operation; Theoretical plate; Mass transfer unit number; Absorption factor

化学工程基础过程中的吸收与精馏是化工中最常见的单元操作过程, 主要用来分离气体和液体 混合物, 涉及到的主要设备就是填料吸收塔与板式精馏塔。它们的主要区别在于填料塔中气液相间 的传质主要是在填料表面上通过气液相间接触来进行的; 而板式塔气液相间的传质主要是通过气相 穿过塔板上的液膜层来完成的。在实际生产过程中, 由于精馏回流的液相流量要比吸收单元操作时 溶剂的用量要小得多, 为了保证运行过程中良好的气液接触传质, 从目前的实际应用来看, 精馏用 板式塔还是比填料塔要多。但是从新技术发展方向分析, 填料塔的发展很快, 因为填料塔中高比表 
面的填料的使用, 使得填料塔相对于板式塔的分离效率要高得多, 可以以 10 倍来计。因此, 大直径 与大高度的板式塔可以被精简到很矮小的尺寸, 这种新技术的革新, 降低了生产工艺中有关分离设 备的安装难度, 设备的投入成本与运行维护费用得到减少 ${ }^{[1-3]}$ 。

对于填料塔的计算主要有塔径与填料层高度的计算, 其中, 填料层高度的计算是关键, 填料塔 塔径的计算与物料的处理量以及填料的性能有关，可通过下式求取塔径:

$$
D=\sqrt{\frac{4 V_{\mathrm{s}}}{\pi \cdot u}}
$$

其中 $V_{\mathrm{s}}$ 为气体的体积流量 $\left(\mathrm{m}^{3} \cdot \mathrm{s}^{-1}\right), u$ 为设计点的空塔气速 $\left(\mathrm{m} \cdot \mathrm{s}^{-1}\right)$, 通常取液泛点气速的 $50 \%-80 \%$, 而液泛点气速可通过填料的通用关联图查得, 即横坐标与纵坐标分别为: $\frac{G_{\mathrm{L}}}{G_{\mathrm{V}}} \cdot\left(\frac{\rho_{\mathrm{V}}}{\rho_{\mathrm{L}}}\right)^{0.5}$, $\frac{u_{\mathrm{f}}^{2} \Psi \Phi}{g} \cdot \frac{\rho_{\mathrm{V}}}{\rho_{\mathrm{L}}} \cdot\left(\frac{\mu_{\mathrm{L}}}{\mu_{\mathrm{W}}}\right)^{0.2}$ 。

式中: $u_{\mathrm{f}}$ 为泛点气速 $\left(\mathrm{m} \cdot \mathrm{s}^{-1}\right), G_{\mathrm{V}} 、 G_{\mathrm{L}}$ 分别为气体与液体的质量流速 $\left(\mathrm{kg} \cdot \mathrm{m}^{-2} \cdot \mathrm{s}^{-1}\right), \rho_{\mathrm{V}} 、 \rho_{\mathrm{L}}$ 分别为 气体与液体的密度 $\left(\mathrm{kg} \cdot \mathrm{m}^{-3}\right), \mu_{\mathrm{L}} 、 \mu_{\mathrm{W}}$ 分别为液体与水的粘度 $(\mathrm{Pa} \cdot \mathrm{s}), \Psi$ 为水的密度与液体密度之比, $\Phi$ 为泛点填料因子 $\left(\mathrm{m}^{-1}\right), g$ 为重力加速度 $\left(\mathrm{m} \cdot \mathrm{s}^{-2}\right)$ 。由横坐标引垂线与填料液泛线相交, 得纵坐标从 而可求得泛点气速。

而填料层高度的计算主要在吸收章节中介绍的, 它采用的是传质单元数法求取, 即填料层高度 等于传质单元高度与传质单元数的乘积; 而板式塔主要是在精馏章节中介绍的, 它涉及一个理论板 的概念, 所谓理论塔板, 是指偏离平衡气体与液体进入一块板并在这块板上进行传质与传热后, 离 开这块板的气体与液体组成间达到平衡时所对应的板。

不管是填料塔还是板式塔都是用来气、液分离的单元设备, 它们均可以用在吸收与精馏单元操 作中。对于填料塔与板式塔达到相同的分离任务时如何相互转换, 则要用到传质单元数与理论板数 之间的关系, 而教材 ${ }^{44,5]}$ 中对二者之间的定量关系表达介绍的较少, 即使有大多也只是给出了一个结 果, 没有推导过程, 这样对学习者的理解会造成一定困难。

下面将传质单元数与理论板数之间的定量关系来加以详细介绍。

\section{1 板式塔中理论板数的求取}

若在给定浓度 $X_{a}, X_{b}$ 范围内, 操作线与平衡线均为直线, 则可用吸收因子法直接计算其理论板 数, 具体计算过程如下:

设气、液相平衡关系为:

$$
Y_{e}=m X_{e}+B
$$

式中 $m$ 与 $B$ 分别为相平衡与直线截距常数。如果 $n$ 代表具体的理论板数, 根据理论板的定义对任一 块理论板则有:

$$
Y_{n}=m X_{n}+B
$$

将 $X_{n}$ 代入操作线方程则有:

$$
Y_{n+1}=\frac{L\left(Y_{n}-B\right)}{m V}+Y_{a}-\frac{L X_{a}}{V}
$$

根据吸收因子的定义有: $A=L / m V$, 即操作线的斜率 $L / V$ 与相平衡线的斜率 $m$ 之比。

则上式可改为:

$$
Y_{n+1}=A\left(Y_{n}-B\right)+Y_{a}-A m X_{a}=A Y_{n}-A\left(m X_{a}+B\right)+Y_{a}
$$

若用 $Y_{a}^{*}$ 代表与进料液相成平衡的气相组成, 则有:

$$
\begin{aligned}
& Y_{a}^{*}=m X_{a}+B \\
& Y_{n+1}=A Y_{n}-A Y_{a}^{*}+Y_{a}
\end{aligned}
$$


于是对第一块板有: $n=1, Y_{1}=Y_{a}$,

$$
Y_{2}=A Y_{a}-A Y_{a}^{*}+Y_{a}=Y_{a}(1+A)-A Y_{a}^{*}
$$

$n=2$ 时有:

$$
\begin{aligned}
Y_{3} & =A Y_{2}-A Y_{a}^{*}+Y_{a}=A\left[Y_{a}(1+A)-A Y_{a}^{*}\right]-A Y_{a}^{*}+Y_{a} \\
& =Y_{a}\left(1+A+A^{2}\right)-Y_{a}^{*}\left(A+A^{2}\right)
\end{aligned}
$$

同理当 $n=N$ 时有: $Y_{n+1}=Y_{N+1}=Y_{b}$

$$
Y_{b}=Y_{a}\left(1+A+A^{2}+\ldots+A^{N}\right)-Y_{a}^{*}\left(A+A^{2}+\ldots+A^{N}\right)=Y_{a} \frac{1-A^{N+1}}{1-A}-Y_{a}^{*} A \frac{1-A^{N}}{1-A}
$$

又由式(6)则有:

$$
Y_{b}=A Y_{N}-Y_{a}^{*} A Y_{a}^{*}+Y_{a}
$$

由图 1 可知: $Y_{N}=Y_{b}^{*}$, 代入式(10)有:

$$
Y_{a}=Y_{b}-A\left(Y_{b}^{*}-Y_{a}^{*}\right)
$$

式(11)代入式(9)则可求出理论板数 $N$ 的关系式如下:

$$
N=\frac{\ln \left[\left(Y_{b}-Y_{b}^{*}\right) /\left(Y_{a}-Y_{a}^{*}\right)\right]}{\ln (A)}
$$

板式塔的理论板数确定后, 如达到相同分离任务要用填料塔来完成时, 则其填料层高度为 $H=$ $H E T P \times N$, 其中 $H E T P$ 为等板高度 $(\mathrm{m})$, 表示分离效果相当于一层理论板时所对应的填料层的高度, $N$ 为理论板数。

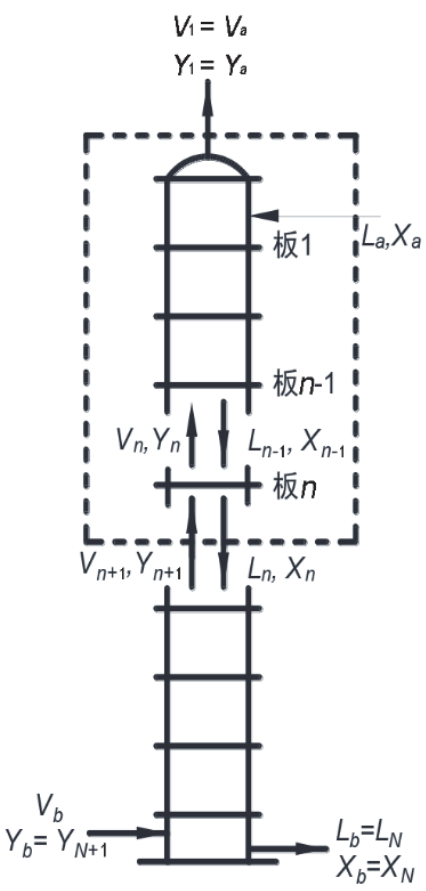

图 1 理论板模型图

\section{2 填料塔中传质单元数的求取}

若在给定浓度 $X_{a}, X_{b}$ 范围内, 操作线与平衡线均为直线, 如图 2 所示, 则可用吸收因子法求总 传质单元数具体计算过程如下:

若气液相平衡关系为:

$$
Y_{e}=m X_{e}+B
$$




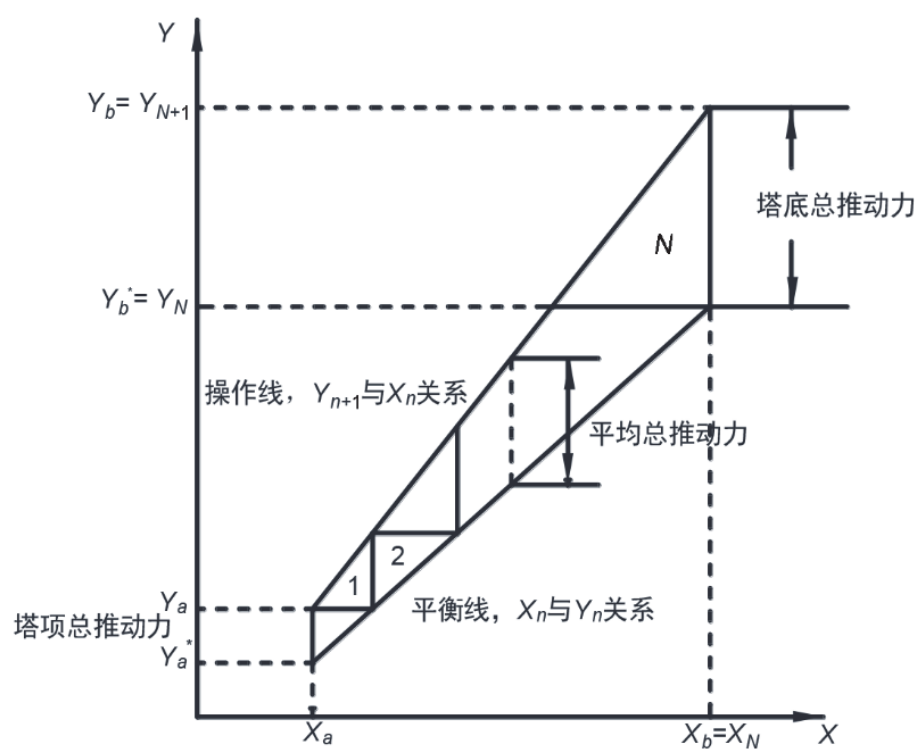

图 2 平衡线、操作线以及总传质推动力示意图

而由物料衡算有:

$$
Y=\frac{L}{G}\left(X-X_{a}\right)+Y_{a}, \text { 得 } X=\frac{V}{L}\left(Y-Y_{a}\right)+X_{a}
$$

根据传质单元数 $N_{\mathrm{OG}}$ 的定义有:

$$
\begin{aligned}
N_{\mathrm{OG}} & =\int_{Y_{a}}^{Y_{b}} \frac{\mathrm{d} Y}{Y-Y_{e}}=\int_{Y_{a}}^{Y_{b}} \frac{\mathrm{d} Y}{Y-m \cdot\left[\frac{V}{L}\left(Y-Y_{a}\right)+X_{a}\right]-B} \\
& =\int_{Y_{a}}^{Y_{b}} \frac{\mathrm{d} Y}{Y\left(1-m \cdot \frac{V}{L}\right)+\left[m \cdot \frac{V}{L} Y_{a}-\left(m X_{a}+B\right)\right]}=\int_{Y_{a}}^{Y_{b}} \frac{Y\left(1-m \cdot \frac{V}{L}\right)+\left[m \cdot \frac{V}{L} Y_{a}-Y_{a}^{*}\right]}{Y\left(Y_{a}\right]} \\
& =\frac{1}{(1-S)} \ln \left(\frac{\left[(1-S) Y_{b}+S y_{a}-Y_{a}^{*}\right]}{\left[(1-S) Y_{a}+S Y_{a}-Y_{a}^{*}\right]}\right)=\frac{1}{(1-S)} \ln \left(\frac{Y_{b}-S\left(Y_{b}-Y_{a}\right)-Y_{a}^{*}}{Y_{a}-Y_{a}^{*}}\right)
\end{aligned}
$$

由于解吸因子 $S$ 与吸收因子 $A$ 互为倒数关系, 由图 2 可知有:

$$
S=\frac{1}{A}=\frac{m V}{L}=\frac{\text { 相平衡线斜率 }}{\text { 操作线斜率 }}=\frac{\left(Y_{b}^{*}-Y_{a}^{*}\right) /\left(X_{b}-X_{a}\right)}{\left(Y_{b}-Y_{a}\right) /\left(X_{b}-X_{a}\right)}=\frac{Y_{b}^{*}-Y_{a}^{*}}{Y_{b}-Y_{a}}
$$

即, $Y_{b}-Y_{a}=A\left(Y_{b}^{*}-Y_{a}^{*}\right)$

代入式(15)中则有:

$$
N_{\mathrm{OG}}=\frac{A}{(A-1)} \ln \left(\frac{Y_{b}-Y_{b}^{*}}{Y_{a}-Y_{a}^{*}}\right)
$$

达到分离任务所需的传质单元数确定后, 则填料塔填料层的高度等于传质单元高度 $H_{\mathrm{OG}}$ 与传质 单元数 $N_{\mathrm{OG}}$ 的乘积, 即:

$$
H=H_{\mathrm{OG}} \times N_{\mathrm{OG}}=N_{\mathrm{G}} \times H_{\mathrm{G}}=N_{\mathrm{L}} \times H_{\mathrm{L}}
$$

式(18)中, 传质单元高度相当于完成一个传质单元分离任务所需要的填料层高度, 或者是使气 相组成变化刚好等于平均传质推动力 $\Delta Y_{m}$ 时所对应的填料层高度。它根据传质推动力和传质系数的 不同, 有总传质单元高度 $H_{\mathrm{OG}}$ 、气相分传质单元高度 $H_{\mathrm{G}}$ 和液相分传质单元高度 $H_{\mathrm{L}}$ 。很显然, 总传 质单元高度 $H_{\mathrm{OG}}$ 对应的是总传质系数, 其推动力为气、液二相的总传质推动力 $Y-Y_{e}$, 其中, $Y$ 为 气相主体的摩尔比浓度, $Y_{e}$ 为与液相主体浓度 $X$ 成平衡时所对应的气相摩尔比浓度; 气相分传质单 元高度 $H_{\mathrm{G}}$ 则对应的是气相分传质系数, 其推动力为气相侧的传质推动力 $Y-Y^{*}$, 其中, $Y$ 为气相主 
体的摩尔比浓度, $Y^{*}$ 为相界面处与液相成平衡时所对应的气相摩尔比浓度; 而液相分传质单元高度 $H_{L}$ 则对应的是液相分传质系数, 其推动力为液相侧的传质推动力 $X^{*}-X$, 其中, $X^{*}$ 为相界面处溶质 的平衡液相摩尔比浓度, $X$ 为液相主体的溶质摩尔比浓度。

显然, 传质单元高度的大小与单位塔截面上气体的处理量、传质性能有关外, 还与填料性能密 切相关，不同传质单元高度之间有如下关系:

$$
H_{\mathrm{OG}}=H_{\mathrm{G}}+\frac{m G}{L} H_{\mathrm{L}}
$$

与传质单元高度相对应的是总传质单元数 $N_{\mathrm{OG}}$ 与分传质单元数 $N_{\mathrm{G}}$ 与 $N_{\mathrm{L}}$, 它们均为无因次量。

$$
N_{\mathrm{OG}}=\int_{Y_{a}}^{Y_{b}} \frac{\mathrm{d} Y}{Y-Y_{e}}, \quad N_{\mathrm{G}}=\int_{Y_{a}}^{Y_{b}} \frac{\mathrm{d} Y}{Y-Y^{*}}, \quad N_{\mathrm{L}}=\int_{X_{a}}^{X_{b}} \frac{\mathrm{d} X}{X^{*}-X}
$$

传质单元数相当于平均传质推动力的个数, 在平均传质推动力下引起混合气体组成的变化。传 质单元数的多少反映了气体分离的难易程度。

如果填料塔完成相同的分离任务要用板式塔来完成, 需要多少块理论板才能完成呢, 要回答这 个问题, 则需要确定传质单元数与理论板数之间的定量关系, 显然, 比较式(12)与式(17)可知传质单 元数与理论板数之间存在如下关系:

$$
\frac{N_{\mathrm{OG}}}{N}=\frac{A \ln (A)}{A-1}
$$

由上式可知, 填料塔完成相同的分离任务用板式塔来完成时所需的理论板数 $N$ 便可决定。此外, 从式(21)可知, 当 $A$ 小于 1 时, $N$ 大于 $N_{\mathrm{OG}}$; 当 $A$ 大于 1 时, $N_{\mathrm{OG}}$ 大于 $N$; 只有当 $A=1$ 时, 即操作 线与相平衡线平行时, 才有 $N=N_{\mathrm{OG}}$ 。

例如: 空气中含有 $\mathrm{SO}_{2}$ 的体积百分比浓度为 $1.6 \%$, 在 1 个大气压下用纯水在横截面积为 $1.5 \mathrm{~m}^{2}$ 和 $3.5 \mathrm{~m}$ 高的填料塔进行洗涤吸收,塔中装填有塑料鞍座填料。已知气体总摩尔流量为 $0.062 \mathrm{kmol} \cdot \mathrm{s}^{-1}$, 液体的总摩尔流量为 $2.2 \mathrm{kmol} \cdot \mathrm{s}^{-1}$, 出口气体中 $\mathrm{SO}_{2}$ 浓度为 $Y_{b}=0.004$ 。若在操作条件下的平衡关系 为 $Y=40 X$, 计算达到分离任务要求的传质单元数 $N_{\mathrm{OG}}$ 以及所需理论板数 $N$, 并进行比较以确定传 质单元高度 $H_{\mathrm{OG}}$ 和等板高度 $H E T P$ 。

根据题意, 隋性气体的摩尔流量为: $V=0.062 \times(1-0.016)=0.061 \mathrm{kmol} \cdot \mathrm{s}^{-1}$

吸收溶剂的摩尔流量为: $L=2.2 \mathrm{kmol} \cdot \mathrm{s}^{-1}$, 相平衡常数 $m=40$, 则吸收因子 $A=L / m V=2.2 / 2.44=0.9$ 。

又因 $Y_{a}=0.004, Y_{b}=0.016$; 由物料衡算有: $V\left(Y_{b}-Y_{a}\right)=L\left(X_{b}-X_{a}\right)$, 得到: $X_{b}=0.003327, Y_{b}=$ $40 \times X_{b}=0.0133$

则由式(20)知传质单元数为:

$$
N_{\mathrm{OG}}=\frac{A}{(A-1)} \ln \left(\frac{Y_{b}-Y_{b}^{*}}{Y_{a}-Y_{a}^{*}}\right)=\frac{0.9}{(0.9-1)} \ln \left(\frac{0.016-0.0133}{0.004-0}\right)=3.54
$$

由传质单元数与理论板数之间的关系式(21)有:

$$
\frac{N_{\mathrm{OG}}}{N}=\frac{A \ln (A)}{A-1}
$$

则,

$$
N=\frac{N_{\mathrm{OG}}(A-1)}{A \ln (A)}=\frac{3.54 \times(0.9-1)}{0.9 \ln 0.9}=3.733
$$

由此可得到达到分离任务所需的理论板数 $N=3.733$, 取整即为 4 块理论板, 由于 $A$ 小于 1 , 所 以所需的理论板数要大于对应的传质单元数 $N_{\mathrm{OG}}$ 。

因给定了填料高度为 $H=3.5 \mathrm{~m}$, 则有等板高度 $H E T P=H / N=3.5 / 4=0.875 \mathrm{~m}$

且对应有传质单元高度 $H_{\mathrm{OG}}=H / N_{\mathrm{OG}}=3.5 / 3.54=0.9887 \mathrm{~m}$

\section{3 结语}

本文采用吸收因子法详细介绍了理论板数与传质单元数的求法, 并由此建立了它们之间的定量 
关系, 可用于填料塔与板式塔达到相同分离任务时, 二类塔之间的相互转化比较。对于工业分离过 程中是选用塔料塔还是选用板式塔提供了一定的依据, 有助于学生在学习吸收与精馏单元操作时的 理解与掌握。

\section{参 考 文 献}

[1] McCable, W. L.; Smith, J. C.; Harriott, P. Unit Operations of Chemical Engineering, 7th ed.; McGraw Hill Education: New York, 2014.

[2] Green, D. W.; Perry, R. H. Perry's Chemical Engineers' Handbook, 8th ed.; McGraw-Hill: New York, 2008.

[3] Judson, K. C. Separation Processes, 2nd ed.; McGraw-Hill: New York, 1980.

[4] Seader, J. D.; Henley, E. J.; Roper, D. K. Separation Process Principles, 3rd ed.; John Wiley \& Sons, Inc.: New York, 2010.

[5] 王志鬼. 化工原理. 第 5 版. 北京: 化学工业出版社, 2018. 